https://doi.org/10.35336/VA-2021-E-39-43

\title{
ASSESSMENT OF THE FREQUENCY AND POSSIBLE RISK FACTORS FOR PAROXYSMAL ATRIAL FIBRILLATION IN THE EARLY POSTOPERATIVE PERIOD AFTER TRANSAPICAL IMPLANTATION OF THE MEDLAB-CT VALVE \\ O.V.Popylkova, S.S.Durmanov, A.B.Voevodin, V.V.Bazylev \\ Federal State Budgetary Institution "Federal Center of Cardiovascular Surgery" of the Ministry of Health of the Russian Federation, Penza, 6 Stasova str.
}

\begin{abstract}
Aim. To study the incidence and possible risk factors for atrial fibrillation (AF) in the early postoperative period after transapical implantation of the first Russian aortic valve (TAP-AVI).

Methods. The study included 118 patients after successful TAP-AVI. Patients with open aortic valve replacement due to dislocation of the prosthesis were excluded, as well as subjects with permanent AF and who died in the early postoperative period. The mean age of the patients was $71.1 \pm 4.9$ years, body mass index $31.1 \pm 5.9 \mathrm{~kg} / \mathrm{m}^{2}$, males $-39.8 \%$, hypertension was present in $93.2 \%$, diabetes mellitus (DM) - in $27.9 \%$, paroxysmal AF - in $12.7 \%$, coronary artery disease (CAD) was in 56.7\%, 8.4\% were smokers. The median follow-up time corresponded to the hospital stay - 9.5 days. To identify cardiac arrhythmias, regular daily ECG recording in 12 leads was initiated from the first day after TAP-AVI. In the presence of palpitations, 24-hour ECG monitoring was performed. Indicators such as age, male gender, DM, history of AF, interatrial block before surgery, $\mathrm{CAD}$, and echocardiographic parameters were studied as possible predictors of AF development in the early postoperative period after TAP-AVI. There were no significant differences in the baseline parameters in patients with AF paroxysms and sinus rhythm.

Results. In the early postoperative period, AF was encountered in 46 (39\%) of patients. New-onset AF occurred in $38(32.2 \%)$ patients. No cerebrovascular event occurred in patients with post-surgery AF. The only statistically significant risk factor for $\mathrm{AF}$ in the postoperative period was $\mathrm{CAD}$ (OR 5.756; 95\% CI 1.009-8.132; $\mathrm{p}=0.048$ ).

Conclusion. In the early postoperative period, the only significant predictor of AF was the presence of documented CAD.
\end{abstract}

Key words: atrial fibrillation; aortic stenosis; transapical aortic valve replacement

Conflict of Interests: nothing to declare

Received: 20.07.2020 Corrected version received: 27.10.2020 Accepted: 02.11.2020

Corresponding author: Popylkova Oxana, E-mail: popylkova@yandex.ru

For citation: Popylkova OV, Durmanov SS, Voevodin AB, Bazylev VV. Assessment of the frequency and possible risk factors for paroxysmal atrial fibrillation in the early postoperative period after transapical implantation of the MedLab-CT valve. Journal of Arrhythmology. 2020;28(E): 39-43. https://doi.org/10.35336/VA-2021-E-39-43.

In the last decade, transapical aortic valve implantation (TAP-AVI) has established itself as an alternative to open aortic valve replacement in patients with a high risk of perioperative complications $[1,2]$.

Specific complications of TAP-AVI include atrial fibrillation (AF) [3-5] and different conducting system disorders in the form of incomplete and complete left bundle branch block, up to a complete atrioventricular (AV) block, requiring the implantation of a permanent pacemaker $[6,7]$.

There are no data on the frequency of AF in the early postoperative period after TAP-AVI of the domestic prosthesis 'MedLab-CT' in the literature.

Aim. To study the incidence and possible risk factors of atrial fibrillation in the early postoperative period after transapical implantation of the first domestic aortic valve 'MedLab-CT'.

\section{MATERIALS AND METHODS}

From October 2015 to December 2019, 173 patients underwent TAP-AVI 'MedLab-CT' by the transapical method. Clinical selection of patients has carried out following ESC/EACT 2017 recommendations for the treatment of val- vular heart disease. The study was of a prospective observational character. The study included 118 patients.

Inclusion criteria:

- successful operation of TAP-AVI 'MedLab-CT' by the transapical method,

- the ability to assess rhythm disturbances before and after surgery.

Exclusion criteria:

- open prosthetic aortic valve implantation due to dislocation of the MedLab-CT prosthesis,

- severe intraoperative complications that led to the death, - permanent atrial fibrillation.

All patients underwent 12-lead electrocardiography (ECG), Holter monitoring, transthoracic and transoesophageal echocardiography (EchoCG), heart multispiral computed tomography with contrast (MSCT), and coronary angiography before surgery.

EchoCG is an important diagnostic method. This method confirms the presence of aortic stenosis and the degree of its calcification; additionally, the function of the left ventricle (LV) is evaluated, and concomitant diseases of the valve or aortic pathology are detected. Transoesoph- 
ageal EchoCG provides an additional assessment of concomitant mitral valve disease and allows for a more accurate calculation of the estimated size of the aortic valve prosthesis and the distance to the mouths of the coronary arteries. MSCT provides additional information about the size and geometry of the aortic root, the ascending aorta and the severity of calcification.

All our patients had severe aortic stenosis with various clinical variants of its manifestation, a preserved ejection fraction without a moderate or pronounced degree of mitral regurgitation. In case of the detection of coronary artery stenosis $>70 \%$ of the diameter in the proximal segments according to the results of coronary angiography in patients with primary indications for TAP-AVI, preliminary/ simultaneous percutaneous coronary intervention with stent implantation of the coronary arteries was performed.

To detect AF in the postoperative period, patients were evaluated daily with regular ECG recordings in 12 leads from the first day after TAP-AVI and 24-hour Holter ECG monitoring after transfer from the intensive care unit and before discharge. Also, a blood test for the determination of blood potassium was performed in the intensive care unit. The potassium level in the blood was in the reference range of 3.5-5.5 mmol/1. The scope of preoperative examination, implantation technique and postoperative management was described in detail above $[1,2,7]$.

The mean age of patients was $71.1 \pm 4.9$ years, including 47 men (39.8\%), with a body mass index of $31.1 \pm 5.9$ $\mathrm{kg} / \mathrm{m}^{2}$. In this cohort, $33(27.9 \%)$ patients had diabetes mellitus, 67 (56.7\%) had coronary heart disease (CHD) $109(93.2 \%)$ had hypertension and 10 (8.4\%) had chronic obstructive pulmonary disease. EuroSCORE was 7.8 [5.1$11.2] \%$ and coronary artery complexity the Syntax Score scale was 5 [0-13] points. Paroxysmal AF before surgery was detected in $15(12.7 \%)$ patients and interatrial block in $23(19.5 \%)$ patients.

The following EchoCG parameters have been recorded: the LV end-diastolic volume was 103.5 [88-125] $\mathrm{ml}$, the LV end-systolic volume was 35.8 [28-49] $\mathrm{ml}$, the LV ejection fraction was $60.5 \pm 11.5 \%$, the thickness of the posterior wall of the $\mathrm{LV}$ was $14.1 \pm 2.5 \mathrm{~mm}$, the interventricular septum was $14.7 \pm 2.4 \mathrm{~mm}$, the anteroposterior size of the left atrium was $39.3 \pm 5.1 \mathrm{~mm}$, and the volume of the left atrium was 76.5[62-95.2] $\mathrm{ml}$.

All patients received anticoagulant therapy (direct and indirect-acting anticoagulants until the target level of the international normalized ratio was reached), anesthesia and anti-inflammatory drugs (if indicated), angiotensin-converting enzyme inhibitors, beta-blockers, antiarrhythmic drugs according to indications and as cardioversion (amiodarone), gastroprotective therapy, and antiplatelet agents (if indicated), and attended physical rehabilitation classes. Upon discharge, all patients were prescribed indirect-acting anticoagulants (warfarin) with recommendations for maintaining an international normalized ratio within 2.0-3.0 for 6 months, followed by switching to monotherapy. The median duration of follow-up in the hospital was 9.5 days.

Statistical analysis

All clinical data of patients were taken from the electronic medical history ('Medialog 7.10 B0119'). Statisti- cal processing of the results was carried out using IBM $®$ SPSS ${ }^{\circ}$ Statistics Version 21 (21.0.0.0). All quantitative variables were checked for the type of distribution using the Kolmogorov-Smirnov criterion, graphically using quantile diagrams, as well as indicators of asymmetry and kurtosis. With a symmetric distribution, the results are expressed as the arithmetic mean and standard deviation $(\mathrm{M} \pm \mathrm{SD})$. If the distribution was not symmetric, then the values are represented by the median $(\mathrm{Me})$ and the interquartile range in the form of the $25^{\text {th }}$ and $75^{\text {th }}$ percentiles. Qualitative data are described using frequencies (n) and fractions (as a percentage).

The risks were assessed using step-by-step multivariate logistic regression analysis. The latter was used to select a set of independent predictors included in the statistical model that affects the dependent variable (the probability of AF in the postoperative period). The data are represented by the achieved significance level (p) and a 95\% confidence interval $(95 \% \mathrm{CI})$. The critical significance level was assumed to be $\leq 0.05$.

\section{RESULTS AND DISCUSSION}

The paroxysmal form of AF in the postoperative period after TAP-AVI 'MedLab-CT' was detected in 46 patients, which required electrical or drug cardioversion in 3 patients. In other cases, spontaneous relief of arrhythmia was noted. During hospitalization, no cerebrovascular events were recorded. In the early postoperative period, hemorrhagic complications were noted in three patients $(2.5 \%)$ in the form of postoperative bleeding (two cases of hemopericardium and one case of haemothorax), which required resternotomy and surgical hemostasis. All of them were observed in a group of patients without documented episodes of AF.The assessment of the estimated risk factors for AF in the early postoperative period was carried out by a step-by-step multivariate logistic regression analysis (Table 1). The only statistically significant risk factor for AF in the postoperative period after TAP-AVI 'MedLab-CT' in our series of observations was the presence of CHD in the patient (OR 5.756; 95\% CI 1.009-8.132; $\mathrm{p}=0.048$ ).

Degenerative aortic stenosis is one of the most frequent valvulopathies in our time, requiring surgical correction in patients with severe aortic stenosis and the presence of clinical manifestations $[8,9]$. Both classical open prosthetics of the aortic valve and TAP-AVI can be accompanied by complications associated with this procedure, including bleeding, vascular damage, thromboembolic complications (in particular, stroke) and arrhythmias [3, 4, 10-13].

First-time AF episodes in the early postoperative period after TAP-AVI attract attention, may require antiarrhythmic and anticoagulant therapy, and may also cause an increase in the duration of hospitalization $[3,4]$. The first time AF was defined by us as stable AF recorded on an ECG during hospitalization or AF more than 30 seconds was recorded on the Holter ECG monitor, detected in patients after TAP-AVI without a previously documented history of arrhythmia.

In some clinical studies, it has been reported that the prevalence of AF before open prosthetics of the aortic valve is $7-43 \%$ and $22-41 \%$ before TAP-AVI $[5,10$, $11,14-16]$. A similar prevalence was described in a meta-analysis which revealed preprocedural AF in $18 \%$ and 
$31 \%$ of patients respectively [17] and in the results of the FRANCE-2 registry preprocedural where AF was diagnosed before TAP-AVI in $25.8 \%$ of patients [18]. In our case, preprocedural AF was diagnosed in $18.6 \%$ (22 people) of patients, while 15 people had a paroxysmal form of AF and 7 (they were excluded from the study) had a permanent form. Thus, AF is observed in a significant proportion of patients before surgery.

According to some of the authors, the frequency of new episodes of AF after open prosthetics of the aortic valve and after TAP-AVI is $31-64 \%$ and $4-32 \%$, respectively $[3-5,10,11,13-16,19]$. Also, according to the results of the FRANCE-2 registry, the first occurrence of AF after TAP-AVI was observed in $6 \%$ of patients [18]. According to M.Vavuranakis et al, who analyzed published articles on the PubMed and Embase databases, the appearance of new AF episodes in the early postoperative period after TAP-AVI reaches from 1 to $32 \%$ [20]. In our study, AF developed in 39.0\% (46 patients), and first-time AF was observed in $32.2 \%$ (38 patients). At the same time, the

\section{Assessment of the estimated risk factors for $A F$ in the} early postoperative period $(n=118)$.

\begin{tabular}{|l|c|c|}
\hline Parameter & $\begin{array}{c}95 \% \text { Confidence } \\
\text { Interval }\end{array}$ & $\mathrm{P}$ \\
\hline Male & $0.114-1.176$ & 0.091 \\
\hline Age & $0.911-1.137$ & 0.758 \\
\hline BMI & $0.919-1.112$ & 0.826 \\
\hline Diabetes mellitus & $0.315-3.407$ & 0.954 \\
\hline Hypertension & $0.384-2.451$ & 0.293 \\
\hline CHD & $1.009-8.132$ & 0.048 \\
\hline COPD & $0.085-3.881$ & 0.568 \\
\hline Atherosclerosis & $0.224-2.017$ & 0.478 \\
\hline CKD & $0.361-3.753$ & 0.799 \\
\hline Preprocedural AF & $0.713-11.425$ & 0.139 \\
\hline Syntax Score & $0.935-1.019$ & 0.270 \\
\hline EuroScore & $0.894-1.074$ & 0.666 \\
\hline Duration of follow-up & $0.966-1.137$ & 0.256 \\
\hline CDS & $0.978-1.024$ & 0.113 \\
\hline EDV & $0.982-1.030$ & 0.643 \\
\hline Posterior wall of the left ventricle & $0.748-1.360$ & 0.956 \\
\hline Interventricular septum & $0.643-1.216$ & 0.449 \\
\hline LA diameter & $0.934-1.199$ & 0.375 \\
\hline LA volume & $0.975-1.017$ & 0.724 \\
\hline Interatrial block & $0.132-1.551$ & 0.207 \\
\hline
\end{tabular}

Note: BMI - body mass index, CHD - coronary heart-disease, COPD - chronic obstructive pulmonary disease, CKD - chronic kidney disease, EuroScore - risk assessment scale for cardiac surgery, Syntax Score - scale for assessing the severity of coronary artery damage when using various tactics of myocardial revascularisation in patients with multivessel coronary artery damage, CDS - course-diastolic-size, EDV- end diastolic volume, AF - atrial fibrillation, LA - left atrial. presence of paroxysmal AF before surgery was not a significant risk factor for AF in the early postoperative period.

There is a risk of overestimating the incidence of firsttime AF in this group of patients. The exclusion of patients with preprocedural AF is often based on a history of previously documented heart rhythm disorders or a short preprocedural screening. But, in $12 \%$ of patients, AF may be asymptomatic [21] and the development of heart rhythm disorders in the postoperative period may be a verification of a previously preprocedural unknown AF [3].Increased knowledge about predisposing factors, optimal post-treatment monitoring, and preventive antiarrhythmic and antithrombotic therapy can reduce the risk of developing secondary complications in relation to new episodes of AF. Despite the wide variety of reasons for the development of AF after surgical correction of aortic stenosis, to date a marker with the maximum prognostic ability has not yet been found.

In the literature, independent predictors of the occurrence of AF after open aortic valve surgery include a preoperative age of more than 70 years, history of AF, elongation of the dispersion of the $\mathrm{P}$ wave and the filtered $\mathrm{P}$ wave, an increase in the thickness of the posterior wall of the LV, the thickness of the interventricular septum $\geq 1.8 \mathrm{~cm}$; a decrease in the maximum blood flow rate in the left atrium appendage, prolonged artificial circulation, a low body mass index, a maximum transvalvular gradient of $\geq 85 \mathrm{~mm}$ $\mathrm{Hg}$, a history of heart failure, as well as left ventricular ejection fraction before surgery and in the early postoperative period $\leq 50 \%[19,22,23]$.

The pathogenesis of arrhythmias during the mini-invasive TAP-AVI procedure is not well-described, although according to some authors, a greater increase in markers of myocardial damage is observed with transapical access, which indicates more extensive myocardial damage compared to transfemoral transcatheter aortic valve implantation [24]. Also, transapical access and preprocedural dilatation of the left atrium are associated with an increased risk of developing new episodes of AF $[3,5]$.

In our study, TAP-AVI was carried out by the transapical method. In our series of observations, possible predictors of the development of AF in the postoperative period after TAP-AVI 'MedLab-CT' included only chronic coronary heart disease $(p=0.048)$ with statistical significance. The mechanisms of the development of AF in uncomplicated CHD and the interaction between AF and coronary perfusion have not been established. But, from the point of view of the probability of developing AF in CHD, according to some authors, the main importance is not the fact of detecting coronary atherosclerosis, but rather the presence of complications of CHD. In patients with coronary artery disease confirmed by coronary angiography but without signs of HF, AF is found in only $0.2-0.8 \%$. In the presence of clinical manifestations of HF, mitral regurgitation, large postinfarction zones, as well as in asymptomatic systolic dysfunction of the left ventricle, the probability of detecting AF increases to $25 \%$ [25-27]. There is also a clear pattern between the duration of the history of coronary heart disease and AF, since the duration of coronary heart disease up to 10 years increases the risk of AF by 2.6 times, and more than 10 years increases the risk of AF by 4 times. 
The prevalence of AF in CHD increases with age: in men over 60 years of age, the incidence of AF increases by 3.6 times compared to middle-aged patients and for women the same indicator increases by 7 times [27].

The main mechanism of AF development in elderly patients with severe degenerative aortic stenosis is chronic pressure overload of the left ventricle and left atrium at later stages of aortic stenosis. As a result, according to Manolis et al. [28], the compliance of the left ventricle decreases and the stiffness of the left ventricle increases, the filling pressure increases, the reserve of coronary blood flow decreases, the tension of the heart walls increases and the sympathetic nervous system and the renin-angiotensin-aldosterone system are activated [28, 29]. A distinctive feature of this process is fibrosis and increased proliferation of connective tissue. Structural rearrangement leads to the fact that there is an electrical dissociation between the muscle bundles, which facilitates the occurrence and maintenance of AF. Over time, tissue remodeling occurs, which contributes to the development and maintenance of AF by changing the atrial structure. Atrial remodeling consists of three components:

1. Electrical remodelling: with the rapid frequency of atrial contractions observed during AF attacks, there is a decrease in the duration of the action potential due to changes in the content of intracellular calcium. Even with a prolonged AF paroxysm after the recovery of the sinus rhythm, electrical remodelling quickly and completely regresses.

2. Contractile remodelling is a decrease in the contractility of the LA because of changes in the content of intracellular calcium against the background of a high heart rate in AF, leading to blood stagnation and thromboembolic complications, in particular stroke.

3. Structural remodelling of tissues occurs after weeks and months. In this case, macro-and microscopic changes occur in the myocardium, which lead to the development of contractile dysfunction and a decrease in heart output.

During transcatheter TAP-AVI, high-frequency stimulation of the ventricles is performed, which can also lead to an imbalance of intracellular calcium, and in the presence of concomitant diseases, structural changes to the myocardium and electrolyte imbalance in the early recovery period after surgery can lead to AF.

Atrial fibrillation is associated with increased mortality, a higher frequency of stroke and other thromboembolic complications, heart failure, decreased quality of life, decreased exercise tolerance, impaired left ventricular function and the development of cognitive dysfunction [25]. According to some authors, new episodes of AF after surgery are also independently associated with adverse events such as stroke, death, and an increase in the length of hospital stay $[4,19,20]$.

According to M.Vavuranakis et al., patients with a history of AF have a higher mortality rate in the early postoperative period after TAP-AVI than individuals with first-time AF paroxysms. According to the same authors, patients with first-time AF paroxysms have at least twice the risk of cerebrovascular events occurring in the subacute phase (1-30 days) after TAP-AVI [20]. According to other authors, the occurrence of asymptomatic AF in the early postoperative period after TAP-AVI is not associated with a deterioration in the short-term prognosis [30].

In our study, no cerebrovascular events were recorded during follow-up, either in the group of patients without a history of AF or in the group with AF. The absence of cerebrovascular events may be associated with anticoagulant therapy with warfarin at that time for 6 months from the first day after TAP-AVI with the achievement of the target INR (2.0-3.0). Currently, the general approach is to double antiplatelet therapy up to 6 months after TAP-AVI except for patients with AF, who are recommended to combine warfarin with one of the antiplatelet drugs (aspirin or clopidogrel).

\section{CONCLUSIONS}

In our series of observations, the paroxysmal form of AF occurred in 46 (39.0\%) patients after TAP-AVI 'MedLab-CT'. AF was detected in 38 (32.2\%) patients for the first time. Cerebrovascular events were not detected in patients with paroxysmal AF in the early postoperative period. The only significant predictor of the occurrence of AF in the early operational period was the presence of coronary heart disease.

\section{REFERENCES}

1. Bazylev VV, Voevodin AB, Shalygina AS. Medium-term results of transcatheter implantation of MedLab-CT aortic valve prosthesis. Russian Journal of Cardiology. 2019;(8): 65-69. (In Russ.). https://doi.org/10.15829/1560-40712019-8-65-69.

2. Bazylev VV, Voevodin AB, Zakharova AS, et al. Early clinical and hemodynamic results of transcatheter aortic valve implantation using the "MedLab-KT" prosthesis. Circulation Pathology and Cardiac Surgery. 2018;22(3): 17-24. (In Russ.). https://doi.org/10.21688/1681-34722018-3-17-24.

3. Amat-Santos IJ, Rodes-Cabau J, Urena M, et al. Incidence, predictive factors, and prognostic value of new-onset atrial fibrillation following transcatheter aortic valve implantation. J Am Coll Cardiol. 2012;59(2): 178-188. https://doi.org/10.1016/j.jacc.2011.09.061.

4. Saxena A, Shi WY, Bappayya S, et al. Postoperative atrial fibrillation after isolated aortic valve replacement: a cause for concern? Ann Thorac Surg. 2013;95(1): 133-140. https://doi.org/10.1016/j.athoracsur.2012.08.077.

5. Motloch LJ, Reda S, Rottlaender D, et al. Postprocedural atrial fibrillation after transcatheter aortic valve implantation versus surgical aortic valve replacement. Ann Thorac Surg. 2012;93(1): 124-131. https://doi.org/10.1016/j. athoracsur.2011.08.078.

6. Letac B, Cribier A, Koning R, et al. Results of percutaneous transluminal valvuloplasty in 218 adults with valvular aortic stenosis. Am J Cardiol. 1988;62(9): 598-605. 7. Popylkova OV, Durmanov SS, Bazylev VV, et al. Cardiac conduction disturbances following transapical «MEDLAB-KT» aortic valve implantation: first results. Journal of Arrhythmology. 2019;26(2): 14-18. (In Russ) https://doi. org/10.35336/VF-2019-2-14-18.

8. O'Brien S, Shahian D, Filardo G, et al. The Society of Thoracic Surgeons 2008 cardiac risk models: part 2 - isolated valve surgery. Ann Thorac.Surg. 2009; 88 (1 Suppl.): 
S23-42. https://doi.org/10.1016/j.athoracsur.2009.05.056. 9. Nikitina TG, Akishbaya MO, Skopin II, et al. Immediate and lateresults of surgical correction of aortic stenosis. Russian Journal of Thoracic and Cardiovascular Surgery. 2007;49(3): 12-8 (In Russ.).

10. Leon MB, Smith CR, Mack M, et al. Transcatheter aortic-valve implantation for aortic stenosis in patients who cannot undergo surgery. N Engl J Med. 2010;363(17): 1597-1607. https://doi.org/10.1056/NEJMoa1008232.

11. Smith CR, Leon MB, Mack MJ, et al. Transcatheter versus surgical aortic-valve replacement in high-risk patients. $N$ Engl J Med. 2011;364(23): 2187-2198. https:// doi.org/10.1056 / NEJMoa1103510.

12. Bagur R, Rodes-Cabau J, Gurvitch R, et al. Need for permanent pacemaker as a complication of transcatheter aortic valve implantation and surgical aortic valve replacement in elderly patients with severe aortic stenosis and similar baseline electrocardiographic findings. JACC Cardiovasc Interv. 2012;5(5): 540-551. https://doi.org/ 10.1016/j.jcin.2012.03.004.

13. Nuis RJ, Van Mieghem NM, Schultz CJ, et al. Frequency and causes of stroke during or after transcatheter aortic valve implantation. Am J Cardiol. 2012;109(11): 16371643. https://doi.org/ 10.1016/j.amjcard.2012.01.389.

14. Roten L, Stortecky S, Scarcia F, et al. Atrioventricular conduction after transcatheter aortic valve implantation and surgical aortic valve replacement. J Cardiovasc Electrophysiol. 2012;23(10): 1115-1122.https://doi.org/ 10.1111/j.1540-8167.2012.02354.x.

15. Appel CF, Hultkvist H, Nylander E, et al. Transcatheter versus surgical treatment for aortic stenosis: patient selection and early outcome. Scand Cardiovasc J. 2012;46(5): 301-307. https://doi.org/ 10.3109/14017431.2012.699636. 16. Nombela-Franco L, Webb JG, de Jaegere PP, et al. Timing, predictive factors, and prognostic value of cerebrovascular events in a large cohort of patients undergoing transcatheter aortic valve implantation. Circulation. 2012;126(25): 3041-3053. https://doi.org/10.1161/CIRCULATIONAHA.112.110981.

17. Meta-analysis of complications in aortic valve replacement: comparison of Medtronic CoreValve, Edwards Sapien, and surgical aortic valve replacement in 8536 patients. Catheter Cardiovasc Interv. 2012;80(1): 128-138. https:// doi.org/10.1002/ccd.23368.

18. Chopard R, Teiger E, Meneveau N, et al. Baseline characteristics and prognostic implications of pre-existing and new-onset atrial fibrillation after transcatheter aortic valve implantation results from the FRANCE-2 Registry. JACC: Cardiovascular Interventions. 2015;8(10) https:// doi.org/10.1016/j.jcin.2015.06.010.

19. Banach M, Goch A, Misztal M, et al. Predictors of paroxysmal atrial fibrillation in patients undergoing aortic valve replacement. J Thorac Cardiovasc Surg. 2007;134(6):
1569-1576. https://doi.org/ 10.1016 / j. jtcvs.2007.08.032. 20. Vavuranakis M, Kolokathis AM, Vrachatis D, et al. Atrial fibrillation during or after TAVI: incidence, implications and therapeutical considerations. Current Pharmaceutical Design. 2016;22(13). https://doi.org/10.2174/138 1612822666151208123050.

21. Flaker GC, Belew K, Beckman K, et al. Asymptomatic atrial fibrillation: demographic features and prognostic information from the Atrial Fibrillation Follow-up Investigation of Rhythm Management (AFFIRM) study. Am Heart J. 2005;149(4): 657-663. https://doi.org/10.1016/j. ahj.2004.06.032.

22. Skopin II, Golukhova YeZ, Iskandaryan ShR. Early postoperative predictors of atrial fibrillations after prosthetic repair of the aortic valve. Journal of Thoracic and Cardiovascular Surgery. 2005;5: 7-11. (In Russ.).

23. [Makarova NV, Durmanov SS, Bazylev VV. Interatrial block: there is an occasion to reflect. Annals of Arrhythmology. 2018;15(2): 112-122 (In Russ.). https://doi.org/ 10.15275/annaritmol.2018.2.6.

24. Rodes-Cabau J, Gutierrez M, Bagur R, et al. Incidence, predictive factors, and prognostic value of myocardial injury following uncomplicated transcatheter aortic valve implantation. J Am Coll Cardiol. 2011;57(20): 1988-1999. https://doi.org/10.1016/j.jacc.2010.11.060.

25. Benjamin EJ, Wolf PA, D'Agostino RB, et al. Impact of atrial fibrillation on the risk of death: the Framingham Heart Study. Circulation. 1998;98: 946-952

26. Naccarelli GV, Varker H, Lin J, et al. Increasing prevalence of atrial brillation and utter in the United States. Am J Cardiol. 2009;104: 1534-1539. https://doi.org/10.1161/01. cir.98.10.946.

27. Balluzek MF, Aleksandrova LN. The frequency and characteristics of the course of coronary heart disease associated with the development of atrial fibrillation. Bulletin of St.-Petersburg University. 2012;2: 3-9. (In Russ.).

28. Manolis AJ, Rosei EA, Coca A. Hypertension and atrial fibrillation: diagnostic approach, prevention and treatment. Position paper of the Working Group 'Hypertension Arrhythmias and Thrombosis' of the European Society of Hypertension. J Hypertens. 2012;30(2): 239-52. https:// doi.org/10.1097/HJH. 0b013e32834f03bf.

29. Heijman J, Voigt N, Nattel S, Dobrev D. Cellular and molecular electrophysiology of atrial fibrillation initiation, maintenance, and progression. Circulation Research. 2014;114(9): 1483-99. https://doi.org/10.1161/CIRCRESAHA.114.302226.

30. Robert R, Porot G, Fichot M, et al. Incidence and predictors of silent AF after transcatheter aortic valve implantation. Archives of Cardiovascular Diseases Supplements. 2018;10(1): 67. https://doi.org/10.1016/j.acvdsp.2017.11.268. 